\title{
SCIENTIFIC Poster Presentation Abstracts
}

which will be deployed and assessed in the next phase of the project.

Conclusion: Structured mammography reporting helps radiologists and surgeons to efficiently and confidently track the management course of their patients. In addition, with the advancement of artificial intelligence, especially deep learning for image classification, clean and labeled image databases can be used for designing computer-assisted decision support systems without significant data preprocessing.

Keywords: Structured Reporting; Mammography; Breast Cancer; Radiology; Artificial Intelligence

\section{• 10.5812/IRANJRADIOL.99153}

\section{Using Kalman Filter to Improve the Accuracy of Diffusion Coefficients in MR Imaging: A Simulation Study}

Sam Sharifzadeh Javidi1, 2, *; Hamidreza Saligheh Rad ${ }^{1,2}$

${ }^{1}$ Physics and Medical Engineering Department, Medicine School, Tehran University of Medical Sciences, Tehran, Iran

${ }^{2}$ Quantitative Medical Imaging Systems Group, Research Center for Molecular and Cellular Imaging, Tehran University of Medical Sciences, Tehran, Iran

${ }^{*}$ Corresponding author: Physics and Medical Engineering Department, Medicine School, Tehran University of Medical Sciences, Tehran, Iran.Email: samjavidi@yahoo.com

\section{Abstract}

Background: The diffusion coefficient of water molecules in different tissues is a biomarker to diagnose and differentiate diseases, tumors, injuries, etc. [1]. Using a motion-sensitizing gradient, it is possible to map the diffusion coefficient into diffusionweighted MRI (DWI) [2]. Although DWI is a good tool for diagnosing, its accuracy in low regime SNR and even especially in high blood consumer organs is in question.

Objectives: We aimed to improve the accuracy of the diffusion coefficient, especially in the presence of noise.

Methods: Diffusion of water molecules at each voxel causes a signal intensity decay that can be measured using a motion-sensitizing gradient. Blood perfusion in capillary network artifacts and measurement noise can affect the real amount of D. In light of considering process noise and measurement noise, Kalman filter is used to cancel perfusion artifacts and measurement noise. Based on the diffusion model ( $S=S 0 \exp (-b D))$, signal intensity was produced several times and a complex Gaussian noise was added to it. Using a Kalman filter, a noise cancelation process was designed to improve the quality of results. The Kalman filter solved a linear problem in the form of state space. Therefore, the diffusion model was rewritten as $\log (\mathrm{S} / \mathrm{SO}) /-\mathrm{b}=\mathrm{D}$ in state space. The Kalman filter predicted the amount of $\mathrm{D}$ and then modified it based on measurements iteratively (Figure 1). Finally, the results of the proposed method and conventional method were compared with true values (Figure 2).

Results: Statistical tests showed that the proposed method was significantly better than the conventional method $(\mathrm{P}<0.01)$. The conventional method caused a bias in the results of DWI due to eliminating micro-vessel perfusion in the capillary network. However, Kalman filter could consider the effects of microvessel perfusion as a process noise and reduce its effects on results. Kalman filter results (Table 1) were compared with true values and the t-test showed no significant alteration ( $P$ $=0.25$ ). Conventional method results were significantly different from true values and Kalman filter results $(\mathrm{P}<0.0001)$.

Conclusion: Diffusion coefficients in the presence of noise and capillary network suffer from bias; however, the proposed method can be used in these situations to improve the quality of DWI images.

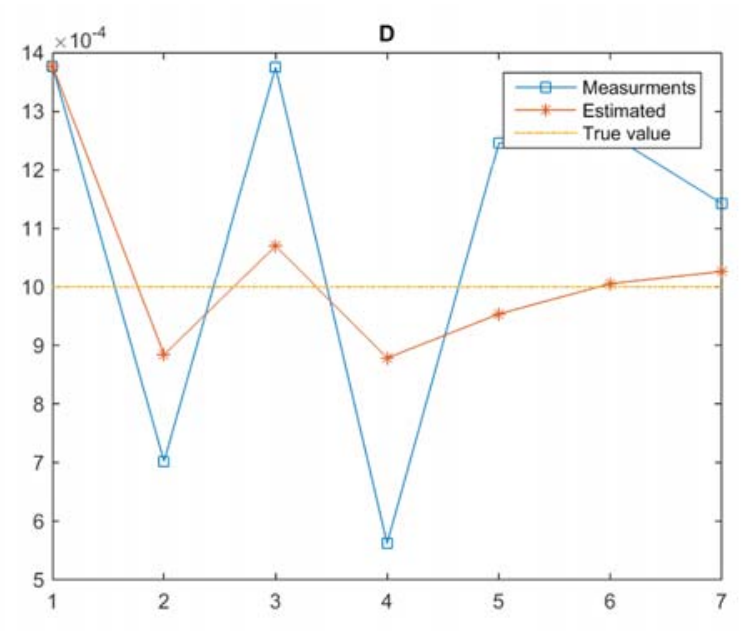

Figure 1. Measurement of D is shown as blue color and varies because of noise, although; Kalman filter improves the result and increases accuracy. 


\section{SCIENTIfic Poster Presentation Abstracts}

Table 1. Statistical Comparison of Kalman Filter and the Conventional Method by t-test; $\mathrm{h}$ is the Decision Based on t-test, 0 Means that Zero Hypothesis is Not Rejected and 1 Means That Alternative Hypothesis is Significant

\begin{tabular}{lcc}
\hline t-test & h & P Value \\
\hline Kalman Filter vs. True values & 0 & 0.2440 \\
\hline Conventional method vs. True values & 1 & $1.8563 \mathrm{e}-48$ \\
\hline Conventional method vs. Kalman filter & 1 & $2.5404 \mathrm{e}-33$ \\
\hline
\end{tabular}

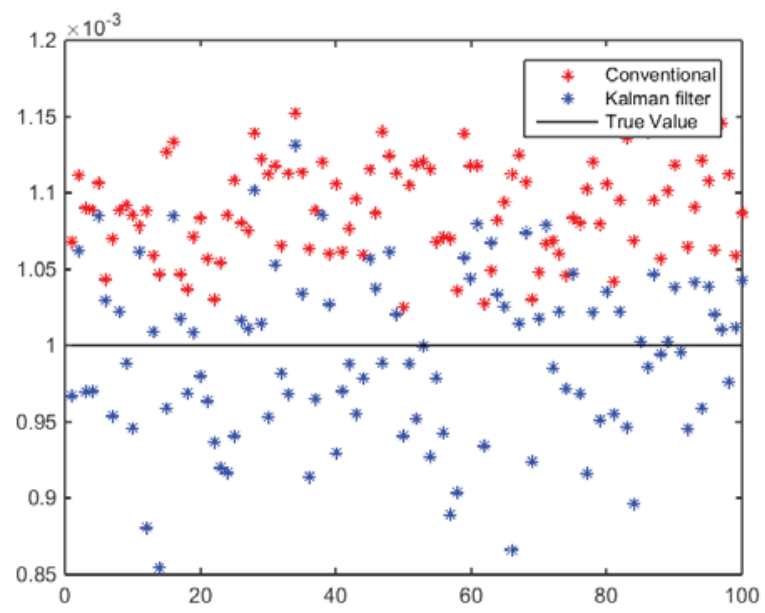

Figure 2. Comparison of the results of Kalman-Filter and conventional method to calculate D. As can be seen, D in the conventional method has a bias.

\section{Bibliography}

1. A. R. Padhani, G. Liu, D. Mu-Koh, T. L. Chenevert, H. C. Thoeny, T. Takahara, A. Dzik-Jurasz, B. D. Ross, M. V. Cauteren, D. Collins, D. A. Hammoud, G. J. Rustin, B. Taouli and P. L. Choyke, "Diffusion-Weighted Magnetic Resonance Imaging as a Cancer Biomarker: Consensus and Recommendations," Neoplasia, vol. 11, no. 2, pp. 102-125, 2009.

2. E. O. Stejskal and J. E. Tanner, "Spin Diffusion Measurements: Spin Echoes in the Presence of a Time-Dependent Field Gradient," Journal of Chemical Physics, vol. 42, no. 1, pp. 288-292, 1965.

\section{-10.5812/IRANJRADIOL.99154}

\section{A Diagnostic Machine Classifier Using Multi-Parametric MRI to Differentiate Benign from Malignant Myometrial Tumors}

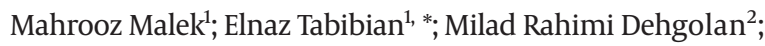

Maryam Rahmani'; Setareh Akhavan'; Shahrzad Sheikh Hasani ${ }^{1}$; Fatemeh Nili ${ }^{1}$; Hassan Hashemi ${ }^{1}$

${ }^{1}$ Tehran University of Medical Sciences, Tehran, Iran

${ }^{2}$ Khaje Nasir Toosi University of Technology, Tehran, Iran

${ }^{*}$ Corresponding author:Tehran University of Medical Sciences, Tehran, Iran.Email: elnaz.tabibian@gmail.com

\section{Abstract}

Background: There are many conditions in medicine that decision making has crucial importance to differentiate between binary diagnoses, such as preoperative discrimination of benign from malignant tumors, e.g. uterine neoplasms. Physicians are not usually able to pool multiple parameters affecting the diagnosis, while "machine learning" techniques, especially "decision trees" with humanreadable results, can process such amounts of data. Previous studies have shown that MRI could be helpful in the differentiation of uterine leiomyosarcoma from leiomyoma.

Objectives: Hereby, for preoperative diagnosis of these tumors and to reduce unnecessary costs and surgeries, we applied a machine classifier using multi-parametric MRI to construct practical diagnostic algorithms.

Methods: A total of 105 myometrial lesions were included in two groups of benign and malignant, according to postoperative tissue diagnosis. Multi-parametric MRI including T1-, T2-, and diffusion-weighted images (DWI) with apparent diffusion coefficient (ADC) map, contrast-enhanced images, as well as MR spectroscopy, were performed for each lesion. Thirteen singular MRI features were extracted from the mentioned sequences. Various combination sets of selective features were fed into a machine classifier (coarse decision-tree) to predict malignant or benign tumors. The accuracy metrics of either singular or combinational models were assessed (Figures 1 and 2). Eventually, two diagnostic algorithms, including a simple decision-tree and a complex one, were proposed using the most accurate models by MATLAB 2017.

Results: Among all singular features, the visual assessment of DWI-ADC restriction, followed by the T2 map, achieved the best accuracies as $96.2 \%$ and $92.0 \%$, respectively. Our final simple decision-tree comprised three features including T2, central necrosis (CN), and DWI-ADC restriction with accuracy of $96.2 \%$, sensitivity of $100 \%$, and specificity of $95 \%$. 